\title{
Proposal for revealing quantum nonlocality via local contextuality
}

\author{
Adán Cabelld* \\ Departamento de Física Aplicada II, Universidad de Sevilla, E-41012 Sevilla, Spain
}

(Dated: June 2, 2018)

\begin{abstract}
Two distant systems can exhibit quantum nonlocality even though the correlations between them admit a local model. This nonlocality can be revealed by testing extra correlations between successive measurements on one of the systems which do not admit a noncontextual model whatever the reduced state of this system is. This shows that quantum contextuality plays a fundamental role in quantum nonlocality, and allows an experimental test of the Kochen-Specker with locality theorem.
\end{abstract}

PACS numbers: 03.65.Ud, 03.67.Mn, 42.50.Xa

Introduction.-One of the most striking aspects of quantum mechanics (QM) is quantum nonlocality; that is, the impossibility of reproducing quantum correlations in terms of classical local hidden variable (HV) theories [1]. In this Letter we will show that there is a fundamentally different way to reveal quantum nonlocality which does not involve classically inexplicable correlations between distant systems. For this purpose, we derive a Bell inequality which is violated by QM. The important point is that the quantum violation occurs even though the correlations between the main system and the auxiliary one can be reproduced with a local model. The obstacle for classical local theories is the state-independent contextuality of one system [2, 3] rather than the correlations between distant systems. This shows that quantum contextuality, a property of quantum systems with more than two states, plays a fundamental role in quantum nonlocality, and provides a different way to experimentally observe quantum nonlocality, valid for any system with $d>2$ states entangled with an auxiliary system with $d$ states. We will illustrate it with an inequality violated if $d \geq 4$, which is particularly simple, but the method can be also applied to any system with $d>2$.

This inequality serves some additional purposes. The first is to point out that, contrary to a common belief (see, e.g., [4]), there is something new to learn about quantum nonlocality from the proofs of the so-called Kochen-Specker (KS) with locality theorem of impossibility of local HV theories [5 9], or "free will" theorem [10, 11], which cannot be learned from other proofs of quantum nonlocality like [1, 12-15]. The second is to elude the criticisms to some recent experiments to test the KS theorem of impossibility of noncontextual HV theories [16 18] with ions [19], neutrons [20], photons [21, 22], and nuclear magnetic resonance systems 23]. The problem of noncontextual HV theories is that the assumption of noncontextuality is not motivated by a physical principle, like locality in Bell inequalities, so one might think that noncontextual theories are physically unplausible [17, 24], since there are classical models reproducing the results of these experiments [25, 26]. A third purpose is to avoid a loophole in these experiments due to the nonperfect compatibility of sequential mea-

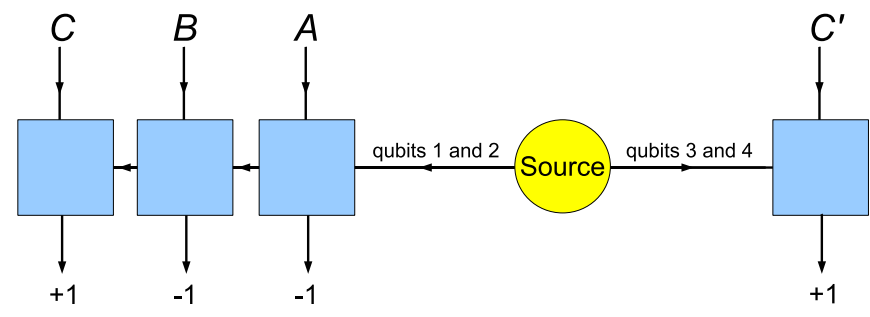

FIG. 1. Scheme of the experiment to reveal quantum nonlocality by local contextuality. Alice performs three compatible dichotomic measurements sequentially, for example, $A, B$, and $C$ on her system (qubits 1 and 2), while Bob performs a single measurement, for example, $C^{\prime}$ on his system (qubits 3 and 4). According to QM, the product of the results of $A$, $B$, and $C$ can be predicted with certainty whatever the state of Alice's system. The experiment also tests the correlations between Alice and Bob's systems. According to QM, in the state (1), the results of $C$ and $C^{\prime}$ are perfectly correlated.

surements [19, 26].

Scenario. - Consider four qubits distributed between two distant locations. Alice has qubits 1 and 2, and Bob has qubits 3 and 4 . In each run of the experiment, Alice performs three successive compatible measurements on the subsystem composed of qubits 1 and 2, and Bob performs a single measurement on the subsystem composed of qubits 3 and 4; as illustrated in Fig. 1. The separation between Alice and Bob's measurements inhibits any communication between Alice's (Bob's) choices of measurements and Bob's (Alice's) results. The four qubits are initially prepared in the state

$$
|\Psi\rangle_{1234}=\left|\psi^{-}\right\rangle_{13} \otimes\left|\psi^{-}\right\rangle_{24},
$$

where $\left|\psi^{-}\right\rangle_{i j}=\frac{1}{\sqrt{2}}\left(|0\rangle_{i} \otimes|1\rangle_{j}-|1\rangle_{i} \otimes|0\rangle_{j}\right)$. On qubits 1 and 2, Alice sequentially measures one of the six sequences: $A B C$ (i.e., first she measures $A$, then $B$, and finally $C$; see Fig. 1), $b a c, \gamma \beta \alpha, A a \alpha, b B \beta$, or $\gamma c C$, where:

$$
\begin{array}{lll}
A=z_{1}, & B=z_{2}, & C=z_{1} z_{2}, \\
a=x_{2}, & b=x_{1}, & c=x_{1} x_{2}, \\
\alpha=z_{1} x_{2}, & \beta=x_{1} z_{2}, & \gamma=y_{1} y_{2},
\end{array}
$$


and $z_{1} x_{2}=\sigma_{z}^{(1)} \otimes \sigma_{x}^{(2)}$, that is, the tensor product of the Pauli matrices $Z$ of qubit 1 and $X$ of qubit 2. Sequential measurements of this type have been recently made on ions [19] and photons [21].

In the state (1), each of the nine observables (2) of qubits 1 and 2 is perfectly correlated or anticorrelated with the corresponding observable of qubits 3 and 4 . In particular,

$$
\begin{array}{lll}
\left\langle B B^{\prime}\right\rangle=-1, & \left\langle C C^{\prime}\right\rangle=1, & \left\langle a a^{\prime}\right\rangle=-1, \\
\left\langle c c^{\prime}\right\rangle=1, & \left\langle\alpha \alpha^{\prime}\right\rangle=1, & \left\langle\beta \beta^{\prime}\right\rangle=1,
\end{array}
$$

where

$$
\begin{array}{llr}
B^{\prime}=z_{4}, & C^{\prime}=z_{3} z_{4}, & a^{\prime}=x_{4}, \\
c^{\prime}=x_{3} x_{4}, & \alpha^{\prime}=z_{3} x_{4}, & \beta^{\prime}=x_{3} z_{4} .
\end{array}
$$

Therefore, in the state (1), the results of $B, C, \ldots, \beta$ can be predicted with certainty from the results of $B^{\prime}, C^{\prime}, \ldots, \beta^{\prime}$, respectively. This prediction is the same regardless of whether $\beta$ is measured in the sequence $\gamma \beta \alpha$ or in the sequence $b B \beta$.

Bell inequality. - Any local HV theory satisfies

$$
\langle\omega\rangle \equiv\langle\chi\rangle+\langle S\rangle \leq 16
$$

where

$$
\langle\chi\rangle \equiv\langle A B C\rangle+\langle b a c\rangle+\langle\gamma \beta \alpha\rangle+\langle A a \alpha\rangle+\langle b B \beta\rangle-\langle\gamma c C\rangle,
$$

and $\langle A B C\rangle$ denotes the average of the product of the outcomes of $A, B$, and $C$ measured in the sequence $A B C$, and

$$
\begin{aligned}
\langle S\rangle \equiv & \left|\left\langle B B^{\prime}\right\rangle_{A B C}\right|+\left|\left\langle B B^{\prime}\right\rangle_{b B \beta}\right|+\left|\left\langle C C^{\prime}\right\rangle_{A B C}\right|+\left|\left\langle C C^{\prime}\right\rangle_{\gamma c C}\right| \\
& +\left|\left\langle a a^{\prime}\right\rangle_{b a c}\right|+\left|\left\langle a a^{\prime}\right\rangle_{A a \alpha}\right|+\left|\left\langle c c^{\prime}\right\rangle_{b a c}\right|+\left|\left\langle c c^{\prime}\right\rangle_{\gamma c C}\right| \\
& +\left|\left\langle\alpha \alpha^{\prime}\right\rangle_{\gamma \beta \alpha}\right|+\left|\left\langle\alpha \alpha^{\prime}\right\rangle_{A a \alpha}\right|+\left|\left\langle\beta \beta^{\prime}\right\rangle_{\gamma \beta \alpha}\right|+\left|\left\langle\beta \beta^{\prime}\right\rangle_{b B \beta}\right|,
\end{aligned}
$$

where $\left\langle B B^{\prime}\right\rangle_{A B C}$ denotes the average $\left\langle B B^{\prime}\right\rangle$ in those events where $B$ is measured in the sequence $A B C$ on qubits 1 and 2 , and $B^{\prime}$ is measured alone on qubits 3 and 4.

Proof: Let us denote by $\hat{B}^{\prime}$ the outcome $(-1$ or 1$)$ the local HV theory assigns to $B^{\prime}$ when no other observable is measured before $B^{\prime}$. Similarly, $\hat{C}^{\prime}$ is the outcome the local HV theory assigns to $C^{\prime}$ when no other observable is measured before $C^{\prime}$. In any local HV theory, $\hat{B}^{\prime}$ and $\hat{C}^{\prime}$ are well defined even though both cannot be simultaneously measured. Therefore, any local HV theory must satisfy the following inequality:

$$
\left\langle A \hat{B}^{\prime} \hat{C}^{\prime}\right\rangle+\left\langle b \hat{a^{\prime}} \hat{c}^{\prime}\right\rangle+\left\langle\gamma \hat{\beta}^{\prime} \hat{\alpha}^{\prime}\right\rangle+\left\langle A \hat{a}^{\prime} \hat{\alpha}^{\prime}\right\rangle+\left\langle b \hat{B}^{\prime} \hat{\beta}^{\prime}\right\rangle-\left\langle\gamma \hat{c^{\prime}} \hat{C}^{\prime}\right\rangle \leq 4,
$$

where the upper bound 4 can be obtained by checking all possible combinations of outcomes $(-1$ or +1$)$ for $A, \hat{B}^{\prime}, \ldots, \gamma$.
Inequality (8) is not directly testable because $\hat{B}^{\prime}$ and $\hat{C}^{\prime}$ (or $\hat{a}^{\prime}$ and $\hat{c}^{\prime}$ ) cannot be measured both in the first place. However, the following sequence of inequalities:

$$
\begin{aligned}
\left|\left\langle A \hat{B}^{\prime} \hat{C}^{\prime}\right\rangle-\langle A B C\rangle\right| \\
\quad \leq\left|\left\langle A \hat{B}^{\prime} \hat{C}^{\prime}\right\rangle-\left\langle A B \hat{C}^{\prime}\right\rangle\right|+\left|\left\langle A B \hat{C}^{\prime}\right\rangle-\langle A B C\rangle\right| \\
\quad \leq\left\langle\left|A \hat{B}^{\prime} \hat{C}^{\prime}-A B \hat{C}^{\prime} \hat{B}^{\prime}{ }^{2}\right|\right\rangle+\left\langle\left|A B \hat{C}^{\prime}-A B C \hat{C}^{\prime 2}\right|\right\rangle \\
\quad=\left\langle\left|A \hat{B}^{\prime} \hat{C}^{\prime}\left(1-B \hat{B}^{\prime}\right)\right|\right\rangle+\left\langle\left|A B \hat{C}^{\prime}\left(1-C \hat{C}^{\prime}\right)\right|\right\rangle \\
\quad \leq 1-\left|\left\langle B B^{\prime}\right\rangle\right|+1-\left|\left\langle C C^{\prime}\right\rangle\right|
\end{aligned}
$$

allows us to see that the term $\left\langle A \hat{B}^{\prime} \hat{C}^{\prime}\right\rangle$ in (8) is lower bounded by experimentally testable quantities,

$$
\left\langle A \hat{B}^{\prime} \hat{C}^{\prime}\right\rangle \geq\langle A B C\rangle+\left|\left\langle B B^{\prime}\right\rangle_{A B C}\right|+\left|\left\langle C C^{\prime}\right\rangle_{A B C}\right|-2 .
$$

Similarly, we can obtain experimentally testable lower bounds for $\left\langle b \hat{a}^{\prime} \hat{c}^{\prime}\right\rangle,\left\langle\gamma \hat{\beta}^{\prime} \hat{\alpha}^{\prime}\right\rangle,\left\langle A \hat{a^{\prime}} \hat{\alpha}^{\prime}\right\rangle,\left\langle b \hat{B}^{\prime} \hat{\beta}^{\prime}\right\rangle$, and $-\left\langle\gamma \hat{c}^{\prime} \hat{C}^{\prime}\right\rangle$. Introducing all of them in (8), we obtain inequality (5).

Quantum violation.-The quantum prediction for the state (1) is

$$
\langle\omega\rangle_{\mathrm{QM}}=18,
$$

which violates inequality (5). This violation is due to a different reason than the violation of previous Bell inequalities [1, 12, 15]. Inequality (5) has two terms: The term $\langle S\rangle$, defined in (7), contains correlations between the two distant systems. In the state (1), all of these correlations are trivial [see Eq. (3)], thus $\langle S\rangle$ takes its maximum value $\langle S\rangle=12$, and inequality (5) becomes

$$
\langle\chi\rangle \leq 4
$$

The term $\langle\chi\rangle$, defined in (6), only contains correlations between successive measurements on one of the local systems (Alice's). Inequality (12) is similar to the inequality in Refs. 2, 19, 21, 23 for noncontextual theories. However, while in Refs. [2, 19, 21, 23], one assumed that if $A$ and $B$ are compatible, then the outcome of $B$ does not depend on whether $A$ has been measured before $B$, inequality (5) holds for any local HV theory, even for those in which a previous measurement of $A$ can change the outcome of $B$.

The difference between the maximum quantum violation and the classical bound is the same for the inequality (5) and the inequality (12). In both cases, the quantum violation occurs because, in QM,

$$
\langle\chi\rangle_{\mathrm{QM}}=6,
$$

since the product of the three operators representing $A$, $B$, and $C$ is the identity $\mathbb{1}$, and the same for $b a c, \gamma \beta \alpha$, $A a \alpha$, and $b B \beta$, while it is $-\mathbb{1}$ for $\gamma c C$. This quantum violation is independent of the state of Alice's system: 
It holds not only when it is in a maximally mixed state [which is the reduced state of qubits 1 and 2 when the state of the four qubits is (1)], but also in any other state. Therefore, neither entanglement nor the reduced state of Alice's system play a role in the violation of inequality (12). Consequently, the role of entanglement in the violation of inequality (5) is marginal. The role of the entanglement of the state (1) is to allow us to convert a test of state-independent quantum contextuality like those in 22, 19, 21, 23] into a Bell test. The only explanation in terms of $\mathrm{HV}$ of the results observed in previous experiments [19, 21, 23] is that the outcomes of the measurements on Alice's system have changed due to previous measurements on Alice's system, as in the models proposed in 25, 26. In the experiment proposed in this Letter, we can test whether this is actually happening by testing wether the expected perfect correlations Bob's system have changed due to these previous measurements. We assume that the outcomes of $A, b$, and $\gamma$ cannot change neither due to previous local measurements (because they are always measured first), nor due to spacelike separated measurements (because we assume locality). However, in the sequence $A B C$, the outcomes of $B$ and $C$ could change by previous local measurements. To detect a contextual behavior in the sequence $A B C$, we measure $A B C B^{\prime}$ (i.e., we measure the sequence $A B C$ on Alice's system, and $B^{\prime}$ on Bob's) in half of the cases, and $A B C C^{\prime}$ in the other half, and then calculate $\left\langle B B^{\prime}\right\rangle_{A B C}$ and $\left\langle C C^{\prime}\right\rangle_{A B C}$.

The entanglement of the state (1) can be replaced by a different kind of entanglement. For example, Alice's fourstate system could belong to a four-system four-level singlet state [27], and a violation of a Bell inequality would occur due to the violation of (12) by performing the same sequential measurements on Alice's system.

Experimental requirements. - As in any Bell experiment, spacelike separation between one observer's choice of measurements and the other observer's result is required if the "upper bound to the speed with which information can be effectively transmitted" is assumed to be the speed of light [10]. We think that there is no fundamental obstacle to satisfy this requirement (see, e.g., [28]), but even an experimental test of inequality (5) without spacelike separation would be important: If the results agree with QM, it would demonstrate the impossibility of contextual explanations of the results on Alice's system under the assumption that no mutual disturbance occurs between the measurements on Alice's system, and the measurements on Bob's, which is a more plausible assumption than the unrestricted noncontextuality assumed in previous experiments 19 23]. The question is whether such an experiment is feasible.

A simple calculation shows that inequality (5) is vio- lated as long as

$$
V>\frac{1}{4}\left(\sqrt{33-2\langle\chi\rangle_{\text {expt }}}-1\right)
$$

where $V$ is the visibility of the prepared states, assuming that, instead of a perfect singlet $\left|\psi^{-}\right\rangle$in (11), we have $\rho=V\left|\psi^{-}\right\rangle\left\langle\psi^{-}\right|+(1-V) \mathbb{1} / 4$, and $\langle\chi\rangle_{\text {expt }}$ is the observed value for $\langle\chi\rangle$. The achieved visibility of two-qubit maximally entangled states with two separated ions is 0.97 , which corresponds to a fidelity $F=0.99$ [29] (assuming that $F=\frac{1}{2} \sqrt{3 V+1}$ ). Then, according to (14), it would be enough to observe $\langle\chi\rangle_{\text {expt }}>4.59$ to violate (5). The achieved violation of the inequality (6) for a two-qubit maximally mixed state is $5.30[19]$. For $\langle\chi\rangle_{\text {expt }}=5.30$, inequality (5) would be violated if $V>0.93$. These results suggest that it is feasible to observe a violation of the inequality (5) with trapped entangled ions. Similar promising results can be obtained by combining the experimental fidelities of pairs of hyperentangled photons (see, e.g., [30]) and the observed violation of the inequality (6) for single photons [21].

Conclusions. - We have shown that there is a different way to experimentally reveal quantum nonlocality based on a violation of a Bell inequality which involves not only correlations between distant systems but also correlations between successive measurements on one of the local systems. The former can be reproduced with local models, the latter cannot be reproduced using noncontextual models. The thing that makes any model that tries to reproduce the trivial correlations with an auxiliary distant system nonlocal is the need of a contextual explanation of the state-independent behavior of one of the systems. This result suggests that the key quantum property behind quantum nonlocality is quantum contextuality, a property of any quantum system with more than two states, rather than only entanglement.

The inequality also provides an experimentally testable version of the KS with locality or "free will" theorem [511], allows us to experimentally exclude models proposed 25,26 to explain the results of some recent experiments 19 23], and avoids the extra assumptions required when dealing with a loophole specific to these experiments 19 , 26].

An experimental violation of this inequality would not only prove the impossibility of local theories and a class of nonlocal theories (those in which the correlations between successive measurements on one system are noncontextual), but would shed new light on the origin of quantum nonlocality, highlighting the importance of quantum contextuality.

The author thanks M. Barbieri, R. Blatt, M. Bourennane, D. P. DiVincenzo, Y. Hasegawa, M. Kleinmann, S. Kochen, B. La Cour, H. Rauch, C. F. Roos, B. Terhal, H. Weinfurter, and A. Zelinger for discussions, O. Gühne for pointing out a hidden assumption in a previous version of the manuscript, J.-A. Larsson, for improving the 
proof of inequality (5), and R. Laflamme for his hospitality at the Institute for Quantum Computing, and acknowledges support from the Spanish MCI Project No. FIS2008-05596, and the Junta de Andalucía Excellence Project No. P06-FQM-02243.

* adan@us.es

[1] J.S. Bell, Physics (Long Island City, N.Y.) 1, 195 (1964).

[2] A. Cabello, Phys. Rev. Lett. 101, 210401 (2008).

[3] P. Badziąg, I. Bengtsson, A. Cabello, and I. Pitowsky, Phys. Rev. Lett. 103, 050401 (2009).

[4] S. Goldstein, D.V. Tausk, R. Tumulka, and N. Zanghì, arXiv:0905.4641.

[5] S. Kochen, letter to A. Shimony in the 1970s, reported in 7], and in [14]. See also [10].

[6] P. Heywood and M.L.G. Redhead, Found. Phys. 13, 481 (1983).

[7] A. Stairs, Phil. Sci. 50, 578 (1983).

[8] M.L.G. Redhead, Incompletness, Nonlocality, and Realism (Oxford University Press, New York, 1987).

[9] H.R. Brown and G. Svetlichny, Found. Phys. 20, 1379 (1990).

[10] J.H. Conway and S. Kochen, Found. Phys. 36, 1441 (2006)

[11] J.H. Conway and S. Kochen, Not. Am. Math. Soc. 56, 226 (2009).
[12] J.F. Clauser, M.A. Horne, A. Shimony, and R.A. Holt, Phys. Rev. Lett. 23, 880 (1969).

[13] D.M. Greenberger, M.A. Horne, and A. Zeilinger, in Bell's Theorem, Quantum Theory, and Conceptions of the Universe, edited by M. Kafatos (Kluwer Academic, Dordrecht, Holland, 1989), p. 69.

[14] D.M. Greenberger, M.A. Horne, A. Shimony, and A. Zeilinger, Am. J. Phys. 58, 1131 (1990).

[15] N.D. Mermin, Phys. Rev. Lett. 65, 1838 (1990).

[16] E.P. Specker, Dialectica 14, 239 (1960).

[17] J.S. Bell, Rev. Mod. Phys. 38, 447 (1966).

[18] S. Kochen and E. P. Specker, J. Math. Mech. 17, 59 (1967).

[19] G. Kirchmair et al., Nature (London) 460, 494 (2009).

[20] H. Bartosik et al., Phys. Rev. Lett. 103, 040403 (2009).

[21] E. Amselem, M. Rådmark, M. Bourennane, and A. Cabello, Phys. Rev. Lett. 103, 160405 (2009).

[22] B.H. Liu et al., Phys. Rev. A 80, 044101 (2009).

[23] O. Moussa, C.A. Ryan, D.G. Cory, and R. Laflamme, Phys. Rev. Lett. 104, 160501 (2010).

[24] J.S. Bell, Found. Phys. 12, 989 (1982).

[25] B.R. La Cour, Phys. Rev. A 79, 012102 (2009).

[26] O. Gühne et al., Phys. Rev. A 81, 022121 (2010).

[27] A. Cabello, Phys. Rev. Lett. 89, 100402 (2002).

[28] W. Rosenfeld et al., Adv. Sci. Lett. 2, 469 (2009).

[29] J. Benhelm, G. Kirchmair, C.F. Roos, and R. Blatt, Nat. Phys. 4, 463 (2008).

[30] M. Barbieri et al., Phys. Rev. Lett. 97, 140407 (2006). 